\title{
VALORACIÓN DE LA IDONEIDAD DIDÁCTICA DE COREOGRAFÍAS DIDÁCTICAS PARA LA EDUCACIÓN ESTOCÁSTICA INFANTIL
}

\section{ASSESSMENT OF THE DIDACTIC SUITABILITY OF DIDACTIC CHOREOGRAPHIES FOR CHILDREN'S STOCHASTIC EDUCATION}

\author{
Gabriela Pilar Cabrera \\ Universidad Nacional de Villa María - Argentina \\ gcabrera@unvm.edu.ar
}

Ana Beatriz Sosa

Directora del Jardín de Infantes Marqués de Sobremonte Adscripta a la Cátedra Matemática y su Didáctica: Instituto Superior de Formación Docente Adoratrices anasosa37@hotmail.com

\section{Resumen}

La educación infantil tiene el reto de constituirse en semilla fértil de una educación estocástica relevante para la formación de una ciudadanía que en contextos de incertidumbre y complejidad sea capaz de la toma las decisiones fundamentada. En respuesta a este desafío, en este artículo se describe, analiza y valora una propuesta de enseñanza para promover la alfabetización estadística, basada en un tipo de coreografía didáctica compleja y enriquecida por los desafíos emergentes del entramado social, natural y tecnológico que habitan los niños y niñas de 4 y 5 años del Jardín de Infantes Marqués de Sobremonte, Córdoba - Argentina; escenario de esta investigación. Se trata de un proceso metodológico de investigación-acción pedagógica que integró los aportes de la Documentación Pedagógica (DP) y de la Teoría de Idoneidad Didáctica (TID) a través de una guía de valoración de la idoneidad didáctica (GVID) que se aplicó a tres coreografías implementadas, obteniéndose un alto grado de idoneidad didáctica global que habilita su recomendación para la alfabetización estadística en edades tempranas y en los primeros años de la escuela primaria. Finalmente, la experiencia de integración de investigación-acción pedagógica, DP y TID potenció el involucramiento activo del equipo docente en la innovación educativa.

Palabras-clave: Educación Estocástica Infantil; Investigación-acción pedagógica; Alfabetización estadística, innovación educativa.

\begin{abstract}
Early childhood education has the challenge of becoming a fertile seed of a relevant stochastic education for the formation of a citizenship that, in contexts of uncertainty and complexity, is capable of making informed decisions. In response to this challenge, this article describes, analyzes and evaluates a teaching proposal to promote statistical literacy, based on a type of complex didactic choreography and enriched by the emerging challenges of the social, natural and technological framework that children inhabit. that children aged between 4 and 5 year old from a Kindergarten in Argentina inhabit; scene of this investigation. It is a methodological process of pedagogical action research that integrated the contributions of the Pedagogical Documentation (DP) and the Theory of Didactic Suitability (TID) through a didactic suitability assessment guide (GVID) that was applied to three choreographies implemented, obtaining a high degree of global didactic suitability that enables its recommendation for statistical literacy at an
\end{abstract}


early age and in the first years of primary school. Finally, the experience of integration of pedagogical action research, DP and TID, promoted the active involvement of the teaching team in educational innovationarly childhood education has the challenge of becoming the seed of a relevant stochastic education for the formation of a citizenry that, in contexts of uncertainty and complexity, is capable of making informed decisions. In response to this challenge.

Keywords: Stochastic Infant Education; Pedagogical action research; Statistical literacy, educational innovation.

\section{INTRODUCCIÓN}

En Argentina en los albores del siglo XXI las prácticas docentes de Matemática en las salas de 4 y 5 años de los jardines de infantes fueron interpeladas por los diseños curriculares jurisdiccionales para el nivel inicial (GOBIERNO DE LA PROVINCIA DE CÓRDOBA, MINISTERIO DE EDUCACIÓN Y CULTURA, 1998) que en aquel momento comenzaron a proponer la enseñanza de la Matemática (GONZÁLEZ y WEINSTEIN, 1998) en la Educación Infantil.

En este contexto, en el año 2000 iniciamos un proceso continuo de investigaciónacción pedagógica cuyo escenario de investigación fue el Jardín de Infantes Marqués de Sobremonte de la ciudad de Villa del Rosario, Córdoba, Argentina; acompañados por el Instituto Superior de Formación Docente (ISFD) Adoratrices, específicamente el Profesorado en Educación Inicial y el Área de Promoción de Calidad Educativa de la Universidad Nacional de Villa María (UNVM).

Como resultante de este proceso de investigación-acción, en este artículo se describe, analiza y valora una propuesta de enseñanza para promover la alfabetización estadística, basada en un tipo de coreografía didáctica compleja y enriquecida por los desafíos emergentes del entramado social, natural y tecnológico que habitan los niños y niñas de 4 y 5 años de un Jardín de Infantes de Argentina; escenario de esta investigación. Se trata de un proceso metodológico de investigación-acción pedagógica que integró los aportes de la Documentación Pedagógica (DP) y de la Teoría de Idoneidad Didáctica (TID) a través de una guía de valoración de la idoneidad didáctica (GVID) que se aplicó a tres coreografías implementadas,

\section{ANTECEDENTES Y MARCO REFERENCIAL}

Al finalizar el siglo XX, Santaló $(1990,1997)$ advertía la presencia exclusiva de una mirada determinista de la Matemática en la escuela y postulaba la necesidad de introducir ideas básicas de Estadística y Probabilidad desde edades tempranas para desarrollar un pensar estadístico y probabilístico. En Vásquez (2020) se evidencia el recorrido significativo de autores que pusieron su esfuerzo en dirección a la promoción 
de la educación estocástica (BATANERO y BOROVCNIK 2016; BATANERO 2019) en la educación infantil siguiendo la premisa de Santaló antes mencionada y se resalta el valor que la educación estocástica tiene para el desarrollo y potenciación de un pensar crítico.

En este sentido, Cabrera, Tauber y Fernández (2020) entienden que la alfabetización estadística y probabilística es el pilar fundamental para el desarrollo de un pensar estadísticamente crítico y que es preciso iniciar esta alfabetización en la educación infantil (CABRERA, 2020). De manera análoga, para Vásquez, Díaz-Levicoy, Coronata y Alsina (2018) la alfabetización estocástica es una "forma de iniciar a los niños [y niñas] en el pensamiento crítico".

Alsina, Cornejo-Morales y Salgado Somoza (2021) "recomiendan que se planteen retos a partir de situaciones cercanas de incertidumbre o experimentos estocásticos (p. 306) y corroboran que los niños y niñas de 4 años empiezan a usar términos estocásticos y expresan grados de creencia para la ocurrencia de sucesos, por medio de una escala cualitativa que va desde lo imposible hasta lo seguro"; esta evidencia se recoge también en Cabrera y Sosa $(2006,2008)$.

Alsina y Vásquez (2017) desarrollan una serie de experiencias contextualizadas para trabajar estadística con niños y niñas de entre 4 a 6 años: “¿Qué vehículos pasan por la rotonda?" y "Estadística con cubos” (p.202-207). Y Vásquez (2020) advierte el valor de la Educación Estocástica en la Educación para el Desarrollo Sostenible a partir de una experiencia didáctica en edades tempranas y convoca a investigadores y docentes a poner en marcha propuestas para promover la conciencia de sostenibilidad que atraviesa todas las dimensiones de la vida contemporánea.

Hasta aquí realizamos una síntesis de investigaciones relevantes que dan cuenta de la importancia y necesidad de promover la educación estocástica en la educación infantil, en un ambiente de aprendizaje en el que la alfabetización estocástica implique que las niñas y niños se integren y a la vez transformen su "ambiente natural, social y tecnológico" (GOBIERNO DE LA PROVINCIA DE CÓRDOBA, MINISTERIO DE EDUCACIÓN y CULTURA, 2020, p.42) en su propio lenguaje y para ello en la investigación que compartimos en este artículo elegimos el camino de las coreografías didácticas.

La metáfora de coreografías didácticas fue acuñada por Oser y Baeriswyl (2001) y permite "modelizar lo visible y no visible de la práctica docente" (CID SABUCEDO, PÉREZ y ZABALZA, 2013, p.271), “es una analogía que posibilita evidenciar la natural 
interconexión que existe entre la performance del artística y el ambiente en el que éste se expresa” (BERAZA y CERDEIRIÑA, 2019, p.212).

Dicho de otro modo, cuando se pone en escena una danza o un cuadro de danzas, cada bailarín tiene una gracia particular que se debe adecuar a los límites que constituyen el escenario, el ritmo y las combinaciones de movimientos, por ejemplo. Esta interpenetración entre coreógrafo, artista y ambiente, plantea una resignificación de la triada didáctica, el rol del profesor y los escenarios educativo (DE LA BARRERA, SOMAVILLA, CHESTA y DE PICCOLI, 2016)

En toda coreografía didáctica deben existir cuatro componentes: la anticipación, la coreografía externa o visible, la coreografía interna del estudiante y el producto o resultado del aprendizaje (ZABALZA, 2017). Cid Sabucedo et al. (2013) sostienen que:

La coreografía externa o visible es el montaje que el profesor hace del contexto de aprendizaje: objetos, espacios, tempos, consignas, demandas, tareas, modos de organización de la clase, [recursos], etc. En tanto, la coreografía interna o invisible son los procesos mentales que provoca esta coreografía externa. Distintas coreografías externas dan lugar a distintas actividades mentales y, por ende, a diferentes procesos de aprendizaje. Una coreografía simple como la lección magistral [clase expositiva] tiene efectos sobre el aprendizaje diferentes a coreografías más complejas y enriquecidas (trabajo en grupo, debates, realización de proyectos, etc.). (p. 271)

Ahora bien, Godino (2021) advierte que para abordar los problemas valorativos que median entre la investigación de diseño y la práctica educativa, la Teoría de Idoneidad Didáctica (TID) (GODINO, 2013) resulta una potente herramienta. Cabe aquí precisar que:

La idoneidad didáctica de un proceso de instrucción se define como el grado en que dicho proceso (o una parte del mismo) reúne ciertas características que permiten calificarlo como óptimo o adecuado para conseguir la adaptación entre los significados personales logrados por los estudiantes (aprendizaje) y los significados institucionales pretendidos o implementados (enseñanza), teniendo en cuenta las circunstancias y recursos disponibles (entorno) (GODINO, 2013, p. 17)

Font (2021) propone preguntas que orientan la aplicación de la guía general de indicadores de idoneidad didáctica (GVID), desarrollado en Godino (2013) para cada una de las seis facetas o dimensiones que componen la idoneidad didáctica global de un proceso de estudio y que contextualizamos para valorar la propuesta de enseñanza basada en un tipo particular de coreografías didácticas que llamamos "macrosituaciones didácticas" (CABRERA y SOSA, 2008, p. 38).

Dicho esto, y siguiendo a Font (2021), para la idoneidad epistémica, las preguntas acerca de si enseñamos una estadística de calidad, es decir, si proponemos procesos relevantes como la resolución de problemas y la modelización estadística; y con todo ello 
-coreografía externa o visible-, ¿logramos que las y los niños aprendan, que desarrollen un nivel de alfabetización estadística adecuado? (idoneidad cognitiva). Sumado a esto, los contenidos que abordamos se corresponden con el currículo de Matemática [Estadística y probabilidad] de Educación Inicial (MINISTERIO DE EDUCACIÓN, CULTURA, CIENCIA y TECNOLOGÍA, Presidencia de la Nación, 2018, GOBIERNO DE LA PROVINCIA DE CÓRDOBA, MINISTERIO DE EDUCACIÓN y CULTURA, 2020), estos contenidos se sitúan en el ambiente natural, social y tecnológico de estos niños y niñas (Idoneidad ecológica). Siguiendo con las preguntas, ¿las tareas que proponemos resultan interesantes para los niños y niñas? ¿tienen sentido? (Idoneidad afectiva), ¿utilizamos recursos temporales, materiales y tecnológicos adecuados? (Idoneidad mediacional) y por último, ¿realizamos una gestión adecuada de las interacciones en la clase que posibilita el abordaje de las dificultades en la resolución de las tareas propuestas? (Idonedad interaccional).

Estas preguntas, además de orientar la aplicación contextualizada de la GVID, permiten evidenciar el diálogo entre la coreografía externa o visible y la interna o invisible. Sumado a esto, se toman las recomendaciones de Beltrán-Pellicer, Godino y Giacomone (2018) para el diseño de indicadores específicos para el logro de la alfabetización estadística en salas de 4 y 5 años

En síntesis, Godino (2021) señala que el sistema de facetas, componentes e indicadores de idoneidad (GODINO 2013; BELTRÁN-PELLICER Y GODINO 2017; BELTRAN-PELLICER, et al 2018; CABRERA y TAUBER, 2019) es una herramienta potente para el proceso de reflexión del profesorado sobre su propia práctica, favoreciendo una dinámica de investigación-acción permanente. En este marco,

\begin{abstract}
La documentación pedagógica es un proceso de indagación que implica el uso del material recogido como medio para la reflexión e interpretación sistemática y democrática, y es llevada a cabo por los educadores en pareja educativa, con el atelierista, o en equipo, aunque también implica procesos individuales, y de reflexión colectiva sobre el trabajo realizado (CIVAROLO, PÉREZ ANDRADA, 2019, p. 6)
\end{abstract}

\title{
METODOLOGÍA
}

La investigación-acción pedagógica (GÓMEZ, 2004) es una investigación de corte cualitativo (AROZA, GODINO y BELTRAN-PELLICER 2016, p.2) a la que incorporamos la GVID y la DP para describir, analizar y valorar tres macrosituaciones didácticas seleccionadas de la colección de coreografías implementadas y publicadas, a saber: Paisaje Urbano (CABRERA Y SOSA, 2006, 2008, p.40-47) y La llegada de la 
Primavera (CABRERA y SOSA, 2006, 2008, p.52-65) para salas de 5 años y La Estación del Tren (SOSA, 2020).

La recolección de datos se obtuvo de la documentación pedagógica a través de registros fotográficos, videos y audios de los modos de resolución que poseen los niños y niñas, registros etnográficos y observaciones de clases. También se realizaron entrevistas, narrativas del equipo docente -tanto individuales como colaborativas-, relatos didácticos, registros autoetnográficos y talleres de reflexión con el equipo docente y estudiantes del profesorado de Educación Inicial del ISFD Adoratrices.

\section{MACROSITUACIONES DIDÁCTICAS}

Las coreografías didácticas "son modelos de práctica educativa que aluden a una combinatoria de arte y ciencia, contexto y compromiso, desde una perspectiva abierta, compleja, creativa e innovadora" (DE LA BARRERA et al. 2016, p.176). En este marco, concebimos un tipo especial de coreografía didáctica, compleja en su dinámica y enriquecida por los desafíos emergentes del entramado social de niños y niñas de 4 y 5 años.

Para expresar la esencia y dinámica de una macrosituación didáctica, elegimos la metáfora de un "splash" que tiñe ese ambiente natural, social y tecnológico en el que habitan niños y niñas y que permite transitar experiencias de aprendizaje que dan respuestas a los desafíos emergentes de ese contexto, transformándolo (CABRERA et al. 2020). Aquí, la danza entre la enseñanza y el aprendizaje estocástico toma un color, un ritmo, un estilo particular que expresamos en el esquema propuesto en la Figura 1.



Figura 1. Macrosituación didáctica: Montaje del ambiente de aprendizaje. 
Vale aquí mencionar que, en su génesis, las macrosituaciones didácticas se anclaron en la dialéctica herramienta-objeto (DOUADY, 1984), dándole un lugar de centralidad al conflicto cognitivo, socio-cognitivo y al error creativo.

\section{Relato didáctico y documentación pedagógica de la Macrosituación didáctica "Paisaje Urbano"}

Contexto, entramado social y toma de decisiones del equipo docente: En el marco de la unidad didáctica "Conociendo nuestro medio", los niños y niñas de las salas de 5 años, en grupos y acompañados por su maestra y algunas madres, recorrieron la manzana que circundaba el Jardín de Infantes. En este recorrido, observaron aquello más relevante y significativo, siendo recurrente en sus observaciones el hecho de que había muchas casas antiguas y otras casas que se estaban arreglando -reciclando-.

Al regresar a la sala se realizó una puesta en común que la docente gestionó, a partir de algunos de los siguientes interrogantes: ¿Cómo son las casas que se encuentran en el barrio? ¿Cuáles son sus características? ¿Por qué son antiguas las casas? ¿Cómo son las casas coloniales? ¿De qué época? ¿Con qué están construidas? ¿Las casas coloniales están recicladas? ¿Qué significa reciclar una casa? ¿Cómo podemos saber cuántas casas antiguas hay?, ¿Cómo podemos comprobar que hay más casas que están arreglando que modernas?

Tiempo para la organización de las tareas emergentes y convergentes para la resolución de las situaciones--problema, gestión colectiva del conocimiento, acuerdos y toma de decisiones: Se realizó nuevamente el recorrido por el barrio organizados en los mismos grupos que en la salida anterior. Cada grupo con el plano del barrio y un cuaderno de registro, debía ubicar cada casa y pintar según las categorizaciones acordadas colectivamente en la sala: rojo-casas moderas, amarillo-antiguas y verde-recicladas (Columna 1, Tabla 1).

Con esta información en la Sala cada grupo escribió la cantidad de cada categoría de casas según lo relevado en el plano, evidenciándose diferentes tipos de registro de cantidades -pictográficos, icónicos y simbólicos- y diagramas simples para la organización de los datos (Columna 2, Tabla 1). En la puesta en común se confrontaron los resultados obtenidos por cada grupo y debido a que no coincidieron algunos y ante el hecho de que todos recorrieron el mismo espacio; se volvió a los planos, se realizó nuevamente el conteo -ahora de forma colectiva-, y cada grupo según correspondió descubrió errores en la registración y/o el conteo y finalmente se consensuaron los datos recolectados. 
A partir del diagrama simple logrado por cada grupo -luego de la reformulación según hubiere correspondido- de manera individual construyeron un gráfico de barras con tres tiras de colores de igual tamaño que cada uno recortó y luego pegó en una hoja que tenía el trazo de una línea recta (Columna 3, Tabla 1). La mayoría, cortó las tiras respetando la relación de magnitud que observaron entre la cantidad de casas antiguas, recicladas y modernas relevadas. Esta información se comunicó a la Municipalidad de la ciudad.

Cabe aquí precisar que nos basados en el Diseño Curricular (GOBIERNO DE LA PROVINCIA DE CÓRDOBA, MINISTERIO DE EDUCACIÓN y CULTURA, 2020) para establecer los contenidos curriculares abordados (Tabla 2) y habiendo advertido la escasa presencia de contenidos de Estadística, nos referenciamos en CABRERA y SOSA (2006, 2008). Esto último se repite las dos macrosituaciones siguientes.

Tabla 1 - Documentación pedagógica del proceso de recolección, organización y presentación de la información recolectada.

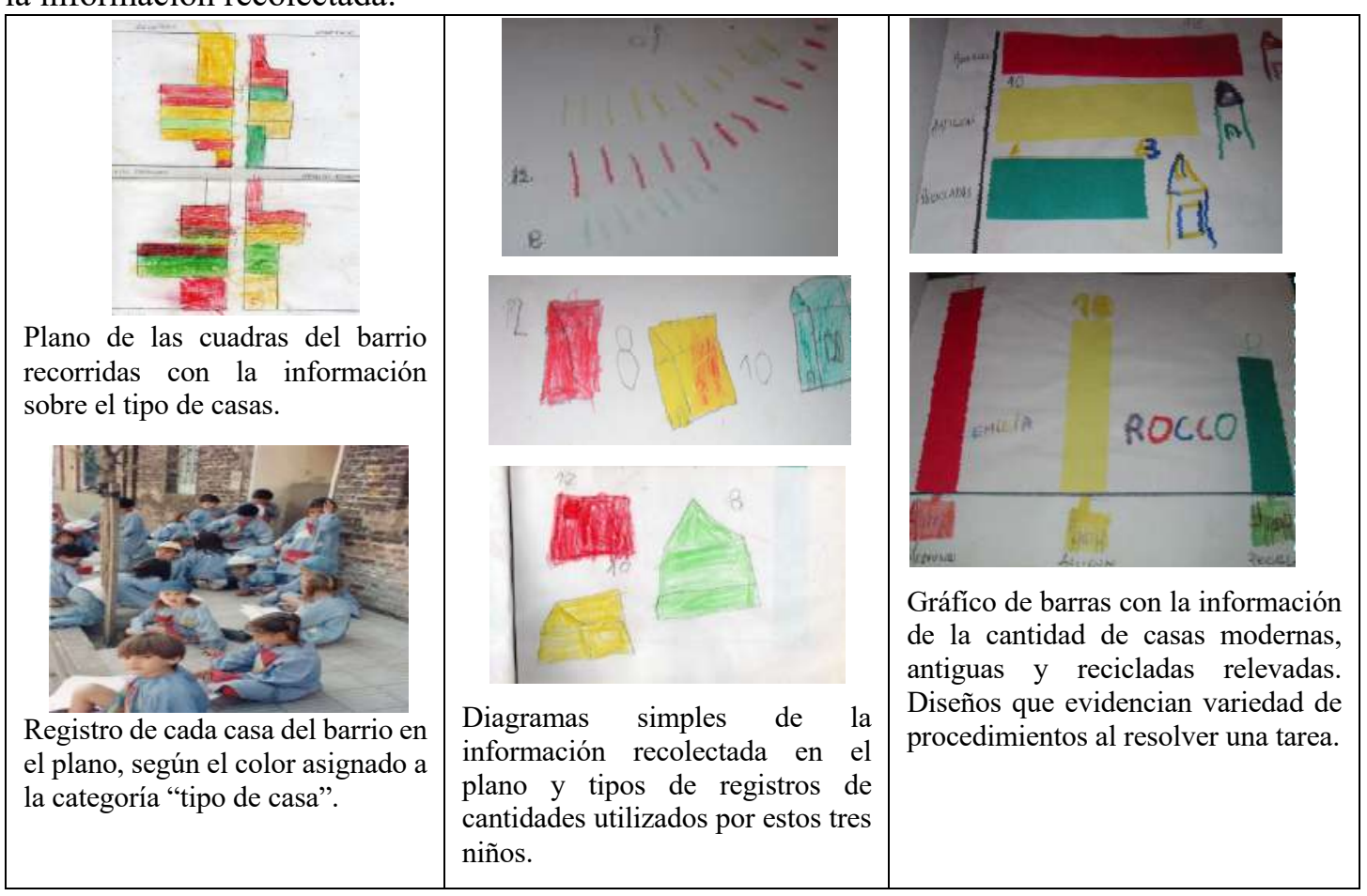

Tabla 2 - Trama de contenidos curriculares desarrollados a partir de la macrosituación Paisaje Urbano.

\begin{tabular}{|l|l|l|}
\hline $\begin{array}{l}\text { Estadística y } \\
\text { Probabilidad }\end{array}$ & $\begin{array}{l}\text { Diseño de un instrumento de recolección de datos. Categorización de los datos. Recolección, } \\
\text { organización y presentación de los datos en diagramas simples y gráficos de barra. Lectura de la } \\
\text { información presentada en tablas, diagramas simples y gráficos de barra. }\end{array}$ \\
\hline $\begin{array}{l}\text { Número y } \\
\text { numeración }\end{array}$ & $\begin{array}{l}\text { Escritura de cantidades: registro pictográfico, icónico y simbólico con apoyo de la banda numérica. } \\
\text { Uso de numero natural para contar, medir y comparar cantidades. Designación oral en situaciones } \\
\text { de conteo. }\end{array}$ \\
\hline $\begin{array}{l}\text { Espacio } \\
\text { Geometría }\end{array}$ & y & $\begin{array}{l}\text { Lectura e interpretación de planos sencillos. Establecimiento de relaciones en el objeto, entre los } \\
\text { objetos y en los desplazamientos. }\end{array}$ \\
\hline $\begin{array}{l}\text { Magnitud } \\
\text { medida }\end{array}$ & yagnitud longitud: establecimiento de comparaciones perceptivas de la longitud de objeto. \\
\hline
\end{tabular}




\section{Relato didáctico y documentación pedagógica de la Macrosituación didáctica "La Estación del Tren"}

Contexto, entramado social y toma de decisiones del equipo docente:

Vale aquí aclarar que durante el ciclo lectivo 2020, en el contexto mundial de la pandemia de Covid-19, las clases en las Salas de 4 y 5 años del Jardín de Infantes Marqués de Sobremonte se desarrollaron a través de un dispositivo sincrónico en la virtualidad, mediadas por WhatsApp a través de audios, fotos y videos. En este marco, el equipo docente consensuó la elección de la estación del tren; un espacio inerte de la ciudad ya que el tren de pasajeros a Buenos Aires dejó de pasar hace muchos años. Este espacio nos pareció potente en cuanto propiciaba un acercamiento de los niños y niñas a este lugar de la ciudad y a su vez lo vislumbramos como propulsor de espacios de juego. En este contexto se propuso la construcción de trenes de juguete y juegos de simulación para la sala de 4 años.

Tiempo para el planteamiento de las situaciones-problema, las tareas emergentes y convergentes para su resolución y "el juego": Con una adivinanza que dice: "Por la vía voy, por la vía vengo. Y en alguna estación, por la vía me detengo" se inició esta escena educativa, en la que niñas y niños pensaron posibles respuestas a esta adivinanza, que dibujaron en su agenda de trabajo. En la siguiente instancia realizamos un juego teatral con títeres y uno de los personajes -Lola-, compartió varios videos que había filmado andando por la ciudad, con motivo de los 200 años de ésta. Allí los dos personajes de la historia, Lola -una niña que va al jardín- y El Marqués de Sobremonte -fundador de la ciudad- se encontraron para hacer un circuito histórico. Esto último, tuvo la intención pedagógica de propiciar la experiencia del afuera a los niños y niñas, ya que en esos momentos en Argentina ocurría el período de confinamiento. En el aula virtual Lola invitó a los niños y niñas a construir el tren para seguir el viaje con el Marqués y dejó la información a modo de enigma en formato de pictograma (Tabla 3).

Tiempo para la organización de las tareas emergentes y convergentes para la resolución de las situaciones-problema, tiempo para las hipótesis y el error creativo: Para descifrar la información proporcionada en el pictograma las niñas y niños debieron leer e interpretar los datos que allí se comunicaban: las formas geométricas y las cantidades de dichas formas que registraron en una tabla de doble entrada proporcionada conjuntamente con el pictograma (Tabla 3). Tradujeron la información del pictograma a la tabla, recortaron la cantidad de cada forma geométrica -según lo anotada en su tabla- y validaron este resultado con el conteo sobre el pictograma; esta actividad la enviaron con un video 
a la docente (Tabla 4).

La caja de luz (Columna 1, Tabla 4) es un recurso atractivo para jugar con formas geométricas, es una caja con un visor transparente dónde se coloca la linterna del celular por dentro y se trabaja con figuras de papeles transparentes en lo posible para lograr el efecto traslúcido; con este recurso armaron el diseño del tren.

Siguiendo en el ritmo de la coreografía, se propuso construir el tren para jugar y usar para ello materiales descartables: cajitas, botones, tapitas, palitos, cartones; ayudados por su familia consiguieron su tren de juguete y su espacio para jugar con este tren. Los diseños se convirtieron en cuadros que se expusieron en el cierre del año lectivo. Una vez más la lectura de los datos, la interpretación de las tablas y el registro de cantidades para crear una obra de arte para la "expo de Arte en Pandemia". Las artes plásticas fueron un vehículo movilizador para plasmar el espacio vivido y transitado, conocieron varios artistas que utilizaban formas geométricas en sus obras de arte. En la Tabla 5, se listan los contenidos curriculares abordados.

Tabla 3 - Documentación pedagógica de la lectura del pictograma y confección de tabla con información para la construcción del tren.

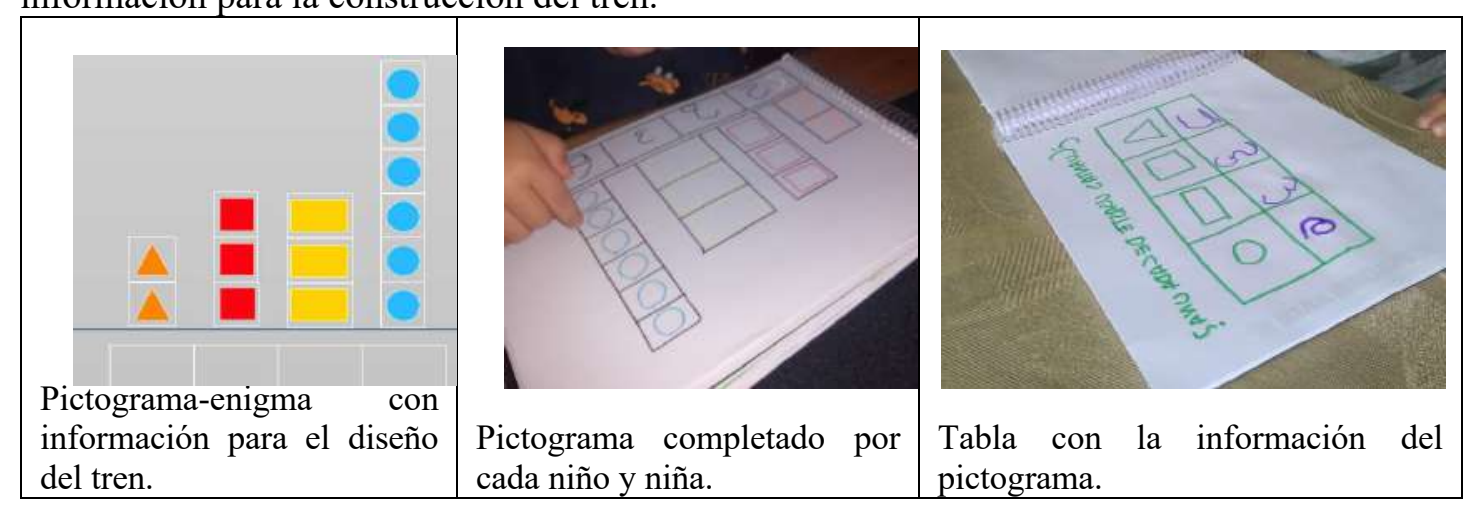

Tabla 4 - Documentación pedagógica de las distintas versiones del tren.

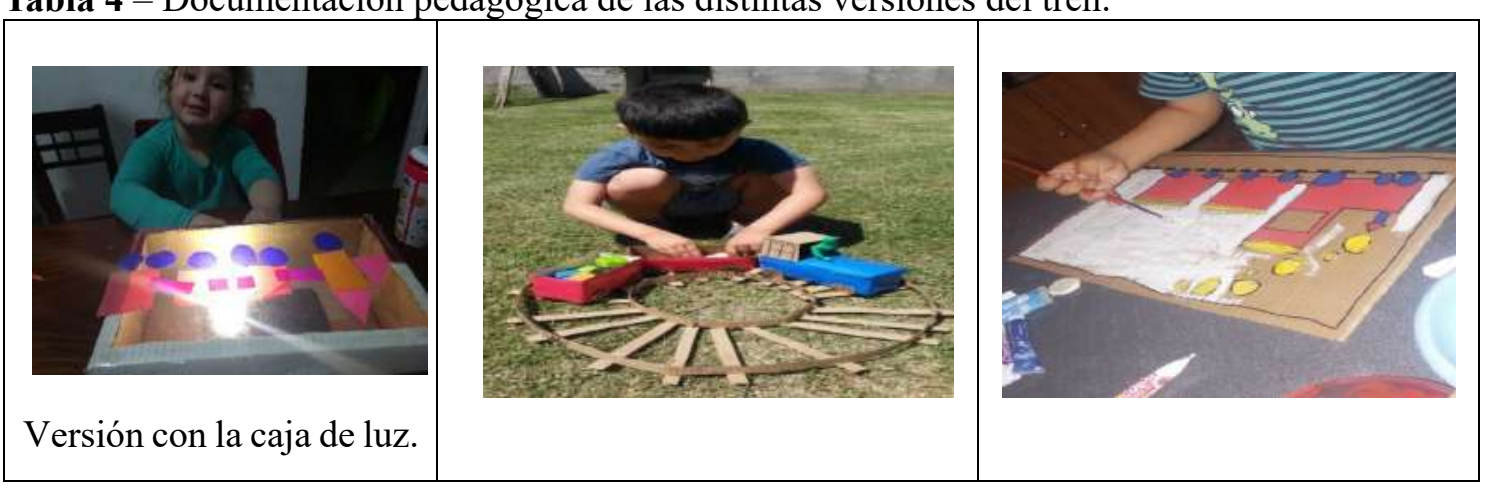

Tabla 5 - Trama de contenidos curriculares desarrollados a partir de "La Estación del Tren".

\begin{tabular}{|l|l|}
\hline Estadística y Probabilidad & $\begin{array}{l}\text { Lectura de información presentada en pictogramas. Presentación de la información en } \\
\text { tablas de doble entrada. Categorización de datos. }\end{array}$ \\
\hline $\begin{array}{l}\text { Número } \\
\text { y numeración }\end{array}$ & $\begin{array}{l}\text { Escritura de cantidades: registro pictográfico, icónico y simbólico con apoyo de la } \\
\text { banda numérica. Uso de numero natural para contar, medir y comparar cantidades. } \\
\text { Designación oral en situaciones de conteo. }\end{array}$ \\
\hline Espacio y Geometría & Reconocimiento global de formas geométricas. Relaciones espaciales en el objeto. \\
\hline
\end{tabular}




\section{Documentación pedagógica de la Macrosituación didáctica "La llegada dela primavera"}

Esta macrosituación fue desarrollada e implementada de manera colaborativa por el equipo docente y estudiantes del Profesorado de Educación Inicial (CABRERA y SOSA, 2006, 2008, p51-65) en salas de 5 años y que por razones de espacio en este documento sintetizamos a través del registro conseguido en la documentación pedagógica (Tablas 6 y Tabla 7).

En la Tabla 6, se muestra la evidencia de los enigmas presentados en formatos de tablas de doble entrada y diagramas de árbol con información de los diseños de flores para confeccionar las guardas, para decorar la Sala con motivo del comienzo de la primavera y la celebración del día del estudiante. En tanto, en la Tabla 7 se evidencia el proceso realizado para consensuar la elección del modelo de collar -que regalarían a madres, tías y/o abuelas en el marco de la conmemoración del día de la madre que se festeja en octubre, tiempo en el cual se desarrollaba esta macrosituación- y que luego confeccionaron con papel maché producidos por ellos mismos. En la Tabla 8, se listan los contenidos curriculares abordados.

Tabla 6 - Documentación de la traducción de enigmas presentados en tablas y diagramas de árbol.

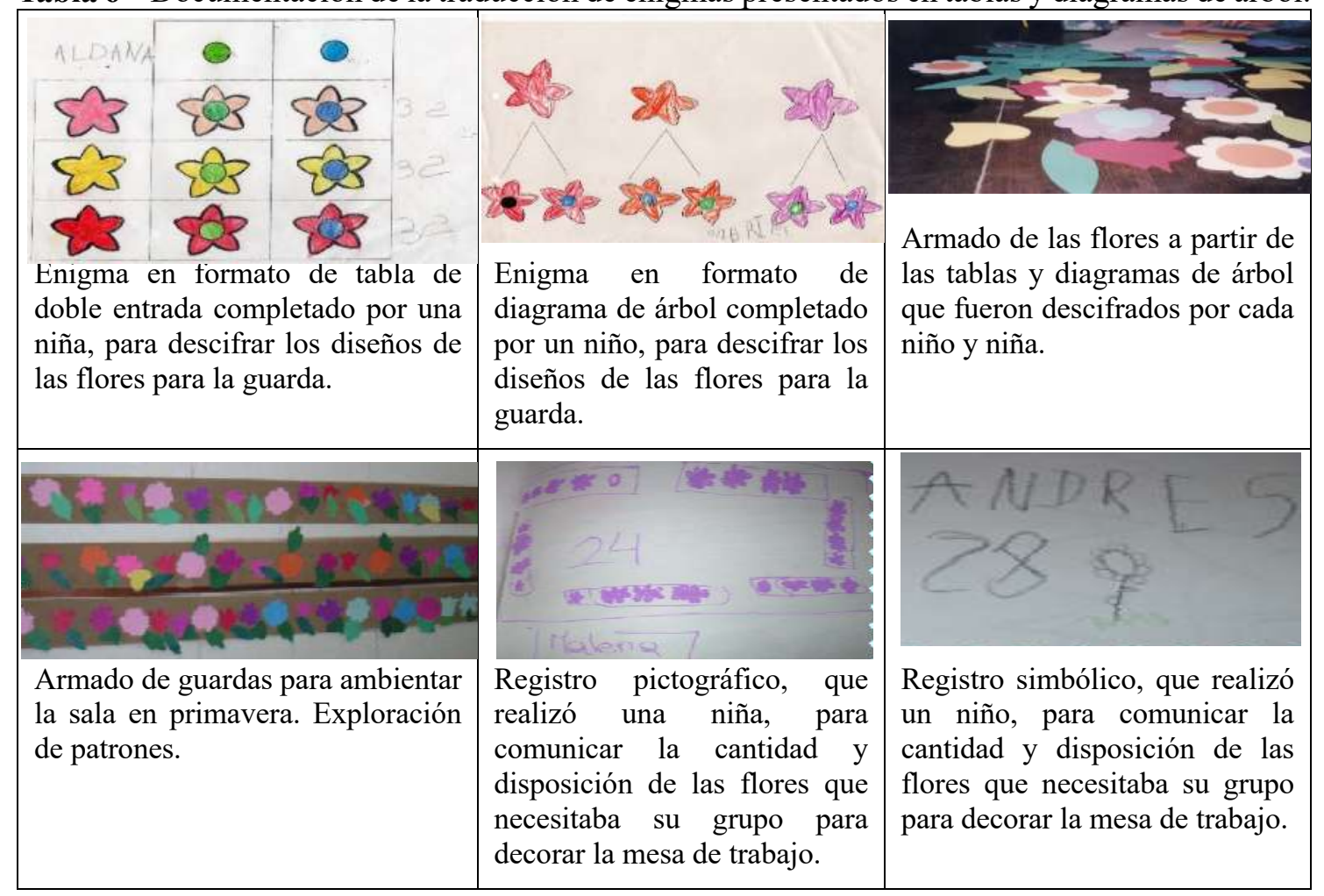


Tabla 7 - Documentación pedagógica del proceso de decisión para el diseño y producción del collar.

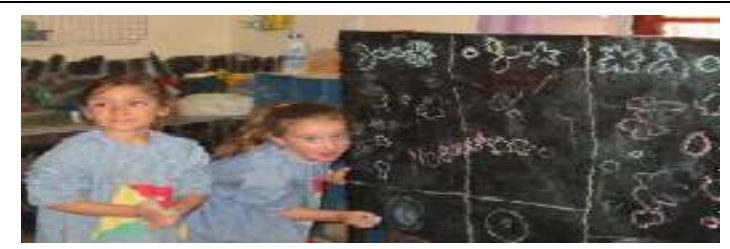

Votación para el diseño de la célula generadora de la serie para el collar.

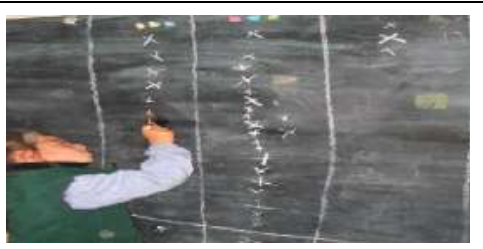

Votación para el diseño de la secuencia de colores para pintar las piezas del collar.

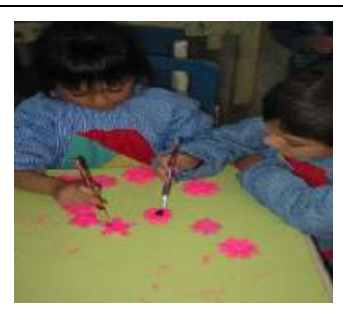

Producción de las piezas del collar según los resultados de la votación.



Armado del collar según la secuencia descifrada del diagrama de árbol.

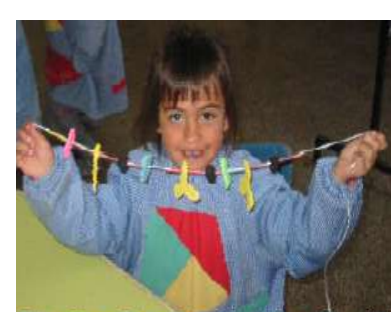

Collar listo para regalar.

Tabla 8 - Trama de contenidos curriculares desarrollados a partir de la macrosituación "La llegada de la Primavera".

\begin{tabular}{|c|c|}
\hline $\begin{array}{l}\text { Estadística y } \\
\text { Combinatoria }\end{array}$ & $\begin{array}{l}\text { Trama de contenidos de combinatoria: Resolución de problemas que impliquen el principio } \\
\text { multiplicativo con el apoyo de tablas de doble entrada y diagramas de árbol sencillos. } \\
\text { Establecimiento de lecturas comparadas entre de tablas de doble entrada y diagramas de árbol. } \\
\text { Lectura y confección de series repetitivas y recursivas a partir de una célula. } \\
\text { Arreglos ordenados de } 2 \text { y } 3 \text { elementos. } \\
\text { Trama de contenidos de Estadística: Diseño de un instrumento de recolección de datos. } \\
\text { Categorización de los datos. Recolección, organización y presentación de los datos en diagramas } \\
\text { simples y gráficos de barra. Lectura de la información presentada en tablas, diagramas simples y } \\
\text { gráficos de barra. }\end{array}$ \\
\hline $\begin{array}{l}\text { Número y } \\
\text { numeración }\end{array}$ & $\begin{array}{l}\text { Escritura de cantidades: registro pictográfico, icónico y simbólico con apoyo de la banda numérica. } \\
\text { Uso de numero natural para contar, medir y comparar cantidades. Designación oral en situaciones } \\
\text { de conteo. }\end{array}$ \\
\hline $\begin{array}{ll}\text { Espacio } & y \\
\text { Geometría }\end{array}$ & Establecimiento de relaciones espaciales en el objeto y entre los objetos. \\
\hline $\begin{array}{l}\text { Magnitud } \\
\text { medida }\end{array}$ & $\begin{array}{l}\text { Iagnitud longitud: uso de unidades de medida no convencionales, como por ejemplo una flor a } \\
\text { avés del cubrimiento y desplazamiento. }\end{array}$ \\
\hline
\end{tabular}

\section{ANÁLISIS Y VALORACIÓN DE LA IDONEIDAD DIDÁCTICA DE LAS MACROSITUACIONES DIDÁCTICAS IMPLEMENTADAS}

En este apartado resumimos el análisis y valoración de las seis facetas que componen la idoneidad didáctica global del proceso de alfabetización estadística mediada por macrosituaciones didácticas y proponemos (BELTRAN-PELLICER et al. 2018, p.528) que "en las circunstancias contextuales fijadas", deberían tenerse en cuenta los criterios e indicadores específicos que exponemos en las Tabla 9, Tabla 10, Tabla 11, Tabla 12, Tabla 13 y Tabla 14 para considerar un alto grado de idoneidad global. Vale recordar que seguimos la guía general GVID propuesta por Godino (2013) y ampliamos algunos de estos indicadores. 
Cabe precisar, que las seis facetas (epistémica, cognitiva, ecológica, afectiva, mediacional e interaccional) se presentan ordenadas, según la lógica que deviene de las preguntas que nos propone Font (2021) y que desarrollamos en el Marco referencial de este documento.

\section{Idoneidad epistémica}

El análisis y valoración de esta idoneidad se realizó para los contenidos curriculares de Estadística y Combinatoria implicados en la alfabetización estadística de niños y niñas de 4 y 5 años de edad. En la Tabla 9, se amplían algunos de los indicadores propuestos de manera general en la GVID (2013).

Tabla 9 - Idoneidad Epistémica.

\begin{tabular}{|c|c|}
\hline Componente & Indicadores \\
\hline $\begin{array}{c}\text { Situaciones } \\
\text { problema }\end{array}$ & $\begin{array}{l}\text { - Selección de una muestra representativa, articulada y contextualizada en el entramado } \\
\text { social en el que habitan los niños y niñas y que da sentido a los siguientes conocimientos: } \\
\text { - Clasificación de la información por dos o tres criterios. } \\
\text { - Recolección, registro y organización de los datos. } \\
\text { - Elaboración, lectura e interpretación de la información presentada en tablas de doble } \\
\text { entrada y diagramas simples. } \\
\text { - Elaboración e interpretación de gráficos estadísticos sencillos: pictogramas y gráficos de } \\
\text { barras. } \\
\text { - Situaciones sencillas de uso de las técnicas de conteo a partir de la lectura de tablas de } \\
\text { doble entrada, diagramas de árbol y procedimientos de conteo y sobre-conteo. } \\
\text { - Situaciones sencillas de arreglos ordenados a partir de la confección de series ordenadas } \\
\text { repetitivas y recursivas. } \\
\text { - Se proponen situaciones que provocan el conflicto-cognitivo y socio-cognitivo. } \\
\text { - Se proponen situaciones que permiten un anclaje significativo de los contenidos a enseñar. }\end{array}$ \\
\hline Lenguajes & $\begin{array}{l}\text { - Se promueve la oralidad a través de la cual las y los niños cuentan, relatan, explican, } \\
\text { disciernen, confrontan y exponen sus ideas. } \\
\text { - Se promueve la evolución de la escritura de cantidades partiendo de un registro } \\
\text { pictográfico hacia el simbólico, con el apoyo de la banda numérica. } \\
\text { - Se presentan en formato de tablas de doble entrada y diagramas de árbol situaciones de } \\
\text { combinatoria sencilla. } \\
\text { - Se presentan en formato de series de figuritas arreglos ordenados sencillos. } \\
\text { - Se comparan información a partir de la lectura de tablas de doble entrada y diagramas de } \\
\text { árbol. } \\
\text { - Se consensuan decisiones colectivas a partir de procesos de votación registrados en tablas } \\
\text { y diagramas simples. } \\
\text { - Se promueve la presentación y lectura de información de gráficos de barras y pictogramas } \\
\text { confeccionados por las y los niños o por las docentes }\end{array}$ \\
\hline $\begin{array}{l}\text { Elementos } \\
\text { regulativos }\end{array}$ & $\begin{array}{l}\text { - Se utiliza un lenguaje claro y atractivo para la presentación de las consignas con apoyo de } \\
\text { variados recursos didácticos. } \\
\text { - Definiciones y procedimientos clara y correctamente enunciados, adaptados al nivel } \\
\text { educativo al que se dirigen. } \\
\text { - Se promueve la generación y negociación de las reglas. }\end{array}$ \\
\hline Argumentos & $\begin{array}{l}\text { - Adecuación de las explicaciones, comprobaciones, demostraciones al nivel educativo al } \\
\text { que se dirigen } \\
\text { - Se promueven momentos de confrontación y validación. } \\
\text { - Se valora positivamente el error creativo. }\end{array}$ \\
\hline Relaciones & $\begin{array}{l}\text { - Se relacionan y articulan de manera significativa los objetos matemáticos puestos en juego } \\
\text { (situaciones, lenguaje, reglas, argumentos) y las distintas configuraciones en que se } \\
\text { organizan. }\end{array}$ \\
\hline
\end{tabular}

Fuente: Adaptación de las autoras. 
implementadas posibilitaron que "enseñemos" una Estadística y Combinatoria de calidad favorecida por una trama de situaciones-problema situadas en contexto, que resultaron significativas para los niños y niñas. Vale mencionar que se propició la iniciación en la modelización estadística, por ejemplo, en Paisaje Urbano se modeló un proceso de recolección de datos -diagramas simples- y comunicación de resultados -gráficos de barra-. En las tres macrosituaciones, las situaciones-problema se plantearon con la intención de generación del conflicto-cognitivo que en los espacios de puestas en común se convirtieron en conflicto socio-cognitivo, dándole de este modo un lugar de centralidad a la argumentación que los niños y niñas desarrollaron a través de la oralidad.

\section{Idoneidad cognitiva}

Para el análisis y valoración de esta idoneidad, nos preguntamos si la coreografía externa de cada una de las tres macrosituaciones implementadas favoreció el aprendizaje de los contenidos curriculares de Estadística y Combinatoria desarrollados. Para ello, en la Tabla 10 ampliamos algunos de los indicadores de la GVID (2013).

Tabla 10 - Idoneidad Cognitiva.

\begin{tabular}{|c|l|}
\hline Componente & \multicolumn{1}{|c|}{ Indicadores } \\
$\begin{array}{c}\text { Conocimientos } \\
\text { previos }\end{array}$ & $\begin{array}{l}\bullet \text { Las niñas y niños disponen de experiencias y conocimientos previos necesarios para el } \\
\text { aprendizaje de los contenidos de Estadística y Combinatoria que se proponen para salas de } \\
\text { 4 y } 5 \text { años y se detallan en la Tabla 9. } \\
\text { - Los significados pretendidos se pueden alcanzar (tienen una dificultad manejable) en sus } \\
\text { diversas componentes. }\end{array}$ \\
\hline $\begin{array}{c}\text { Aula } \\
\text { heterogénea }\end{array}$ & $\begin{array}{l}\text { - Se incluyen variedad actividades de distintos niveles de complejidad. } \\
\text { - Se incluyen variedad de recursos didácticos para los niveles de complejidad diferentes. } \\
\text { - Inclusión }\end{array}$ \\
\hline Aprendizaje & $\begin{array}{l}\text { - La evaluación se desarrolla de manera continua con el soporte de rúbricas diseñadas para } \\
\text { dar cuenta del estado del aprendizaje de cada niño y niña en relación con los contenidos de } \\
\text { Estadística y Combinatoria objeto de enseñanza. }\end{array}$ \\
\hline
\end{tabular}

Fuente: Adaptación por las autoras.

Ante una situación-problema que implica la recolección de datos, por ejemplo, en el relevamiento de la cantidad de casas modernas, antiguas y recicladas en Paisaje Urbano (Tablas 1) es necesario que niñas y niños dispongan del registro pictográfico de pequeñas cantidades, en caso contrario si se encuentran en una etapa de registro idiosincrásico esta actividad resultará un obstáculo que no podrá resolver. Por el contrario, a partir de esta actividad se favorece la evolución del registro de cantidades pictográfico al simbólico de cantidades menores que 10, consiguiéndose con el apoyo de la banda numérica, la cardinalización. Es de destacar que la puesta en común de los modos de registros de los distintos grupos, esa heterogeneidad de modos de escritura promueve esta evolución.

En el caso de La estación del tren, el niño y la niña tendrán que disponer de 
experiencias previas de conteo oral con el soporte de la banda numérica, para la lectura del pictograma (Tabla 3) que se les presenta como enigma para la construcción del tren y la escritura del cardinal correspondiente a cada forma geométrica indicada en la tabla de doble entrada. En tanto, para descifrar el enigma presentado a través de tablas de doble entrada y diagramas de árbol a dos criterios de clasificación en La llegada de la Primavera (Tabla 6 y Tabla 7), las niñas y niños requieren experiencias previas de lectura de tablas a un criterio de clasificación, por ejemplo; valen también calendarios y agendas semanales. Es evidente la recurrencia del registro de cantidades y del conteo oral en todas las actividades implementas.

La dinámica de las macrosituaciones y la documentación pedagógica que se realiza habitualmente en la Sala, promueve y potencia el proceso de evaluación continua porque se ancla justamente en las evidencias que niñas y niños muestran al resolver cada una de las tareas emergentes y convergentes para la resolución de las situacionesproblema.

\section{Idoneidad ecológica}

El análisis y valoración de esta idoneidad implica que nos preguntemos acerca de si los contenidos curriculares que desarrollamos a través de las macrosituaciones implementas, se adecuan a los documentos curriculares que usamos de referencia y para ello se siguen los indicadores de la Tabla 11.

Tabla 11 - Idoneidad Ecológica.

\begin{tabular}{|c|c|}
\hline Componente & Indicadores \\
\hline Adaptación al currículo & $\begin{array}{l}\text { - Los significados, su implementación y evaluación se corresponden con } \\
\text { las directrices curriculares. }\end{array}$ \\
\hline $\begin{array}{c}\text { Apertura hacia } \\
\text { la innovación didáctica }\end{array}$ & - Innovación basada en la investigación y la práctica reflexiva \\
\hline $\begin{array}{l}\text { Conexiones Intra e inter- } \\
\text { Disciplinares }\end{array}$ & $\begin{array}{l}\text { - Los significados se relacionan con otros contenidos intra e } \\
\text { interdisciplinares como condición necesaria para la enseñanza de la } \\
\text { Estadística, Probabilidad y Combinatoria en la educación infantil. }\end{array}$ \\
\hline
\end{tabular}

Fuente: Godino (2013)

Vale aclarar que en los documentos curriculares antes indicados se proponen exclusiva y explícitamente contenidos curriculares referidos a los Ejes: Número y Numeración, Geometría y Espacio, Magnitud y Medida con algunas orientaciones didácticas centradas en el juego y en NAP, 2004; DCJ de la Provincia de Córdoba, no se contempla el Eje: Estadística y Probabilidad. Sólo en IPA 2030 (p.2) se plantea "Interpretación de la información socialmente significativa para el niño, expresados en listas" y este contenido se indica en el Área de contenido “Tratamiento de la información”. Si bien, el registro de cantidades se asocia directamente con el proceso de recolección y 
registro de datos y fueron recurrentes en las tres macrosituaciones implementadas, fue una decisión del equipo docente promoverlos a través de situaciones-problema que abordaran contenidos de Estadística.

Dicho esto, el equipo docente tomó la decisión de referenciar los contenidos curriculares de Estadística y Combinatoria, en la literatura referida a alfabetización estadística en la Educación Infantil ya citada en el marco referencial de este artículo.

Sumado a esto, vale resaltar que estas coreografías didácticas se concibieron en el marco de las Ciencias Sociales, Naturales y Tecnología, la Educación Artística, la Educación Física y Lengua y Literatura, desafíos que emergieron del ambiente natural, social y tecnológico de los niños y niñas los que les dan sentido a conexiones intra e inter disciplinares.

\section{Idoneidad Afectiva}

El análisis y valoración de esta idoneidad implica que nos preguntemos si las tareas que proponemos resultan interesantes para los niños y niñas. Para ello, en la Tabla 12 ampliamos algunos de los indicadores propuestos de manera general en la GVID (2013).

Tabla 12 - Idoneidad Afectiva.

\begin{tabular}{|c|c|}
\hline Componente & Indicadores \\
\hline $\begin{array}{c}\text { Intereses y } \\
\text { necesidades }\end{array}$ & $\begin{array}{l}\text { - Se construyen tareas que tienen interés para los niños y niñas. } \\
\text { - Se proponen situaciones en los que las tablas, gráficos y la recolección de datos posibilita la } \\
\text { resolución de situaciones del ambiente natural, social y tecnológico de los niños y niñas. }\end{array}$ \\
\hline Emociones & $\begin{array}{l}\text { - Se planifican momentos en los que se manifiestan las emociones ante las situaciones } \\
\text { propuestas. } \\
\text { - Se promueve la autoestima, promoviendo la confianza en la resolución de los problemas a } \\
\text { partir de las heurísticas y procedimientos personales. }\end{array}$ \\
\hline Actitudes & $\begin{array}{l}\text { - Se relacionan las emociones positivas con las actitudes positivas hacia la Estadística y con la } \\
\text { resolución exitosa de tareas, fomentando la reflexión emocional de las niñas y niños. } \\
\text { entusiasmo, etc. }\end{array}$ \\
& $\begin{array}{l}\text { Se favorece la argumentación a partir de la oralidad, en situaciones de igualdad; el argumento } \\
\text { se valora en sí mismo y no por quién lo dice. }\end{array}$ \\
\hline Se favorece la confianza en las posibilidades personales de resolución de los problemas.
\end{tabular}

Fuente: Adaptación propia de las autoras.

En La llegada de la primavera, los niños y niñas se sintieron muy entusiasmados con el proceso de diseño y producción del collar-regalo para la mamá, la tía, la abuela o la persona que eligieran -ya que el mes de octubre en Argentina se celebra el día de la madre- y la ambientación de la Sala para celebrar dicho día. Esto favoreció el abordaje de la lectura de tablas de doble entrada y diagramas de árbol en contexto de resolución de un problema de combinatoria y de arreglos ordenados, también la implicación en el proceso consensuado por medio de una votación colectiva para acordar el diseño del collar. 
Esta experiencia se replica año tras año, en otros contextos como por ejemplo: guardas de otoño, banderines para festejos, guardas de vinchas y utensilios aborígenes, combinación de menús para el taller de alimentación saludable, combinación de menús de cuentos en la biblioteca, menús literarios.

\section{Idoneidad Mediacional}

El análisis y valoración de esta idoneidad implica que nos preguntemos acerca de la adecuación y potencia de los recursos temporales, materiales y tecnológicos que utilizamos en las macrosituaciones implementadas. Para ello, en la Tabla 13 ampliamos algunos de los indicadores propuestos de manera general en la GVID (2013).

Tabla 13 - Idoneidad Mediacional.

\begin{tabular}{|c|l|}
\hline Componente & \multicolumn{1}{|c|}{ Indicadores } \\
\hline Recursos Materiales & $\begin{array}{l}\text { - Uso de materiales manipulativos e informáticos que permiten enunciar buenas } \\
\text { situaciones, lenguajes, procedimientos, argumentaciones. } \\
\text { - Las ideas estadísticas son contextualizadas y motivadas usando situaciones, modelos } \\
\text { concretos y visualizaciones. }\end{array}$ \\
\hline $\begin{array}{c}\text { Número de } \\
\text { estudiantes, horarios y y } \\
\text { condiciones del aula }\end{array}$ & $\begin{array}{l}\text { - El número de estudiantes es razonable. } \\
\text { - El tiempo dedicado a las tareas es apropiado. } \\
\text { - La Sala es adecuada al número de niños y niñas y está ambientada estéticamente. }\end{array}$ \\
\hline Tiempos & $\begin{array}{l}\text { - Adecuación de los significados pretendidos /implementados al tiempo disponible. } \\
\text { - Se invierte tiempo en el planteamiento de la situación-problema contextual. } \\
\text { - Se invierte tiempo en que los niños y niñas evidencien las experiencias previas y } \\
\text { planteen sus hipótesis. }\end{array}$ \\
& $\begin{array}{l}\text { - Se invierte tiempo en la gestión colectiva del conocimiento. } \\
\text { - Se invierte el tiempo en la organización y la generación de acuerdos. } \\
\text { - Se invierte el tiempo en las síntesis y toma de decisiones. } \\
\text { - Se invierte el tiempo en la comunicación y devolución de los resultados y decisiones } \\
\text { a la comunidad. }\end{array}$ \\
\hline
\end{tabular}

Fuente: Adaptación propia de las autoras

El Jardín de Infantes Marqués de Sobremonte, escuela pública estatal, funciona en dos turnos, tiene 8 salas estéticamente ambientadas para la edad de las niñas y niños. En cada Sala se trabaja con no más 30 estudiantes. Se organizan espacios y tiempos flexibles y promueven los agrupamientos múltiples para el trabajo colaborativo. Se aprovechan al máximo los recursos materiales y se gestiona una adecuada organización de los mismos $\mathrm{y}$ de los tiempos.

Vale aquí resaltar que la dinámica de las macrosituaciones didácticas contempla desde su diseño, los tiempos propuestos como indicadores específicos para valorar esta idoneidad y que consecuentemente, se promueven y respetan como se observa en los relatos didácticos de las tres macrosituaciones implementadas.

\section{Idoneidad Interaccional}

El análisis y valoración de esta idoneidad implica que nos preguntemos acerca de 
la gestión colectiva del conocimiento en la clase a partir de las macrosituaciones implementadas. Para ello, en la Tabla 14 ampliamos algunos de los indicadores propuestos de manera general en la GVID (2013).

Tabla 14 - Idoneidad Interaccional.

\begin{tabular}{|c|c|}
\hline Componente & Indicadores \\
\hline $\begin{array}{l}\text { Interacción entre } \\
\text { docentes, niños y } \\
\text { niñas, familias }\end{array}$ & $\begin{array}{l}\text { - La docente presenta los desafíos (situación-problema), debidamente contextualizados, de } \\
\text { - Sodo que resulte significativo para los niños y niñas. } \\
\text { los silencios, sus expresiones faciales, sus preguntas, se hace un juego de preguntas y } \\
\text { respuestas adecuado, etc.) } \\
\text { - Se busca llegar a consensos con base al mejor argumento. } \\
\text { - Se usan diversos recursos didácticos para implicar y captar la atención de niñas y niños. } \\
\text { - Se facilita la inclusión de los alumnos en la dinámica de la clase y no la exclusión }\end{array}$ \\
\hline $\begin{array}{l}\text { Interacción entre } \\
\text { niños y niñas }\end{array}$ & $\begin{array}{l}\text { - Se promueve el conflicto-socio cognitivo en la gestión colectiva del conocimiento, } \\
\text { - Se favorece el diálogo y comunicación entre los estudiantes. } \\
\text { - Se favorece la inclusión en el grupo y se evita la exclusión. }\end{array}$ \\
\hline Autonomía & $\begin{array}{l}\text { - Se contemplan momentos en los que los estudiantes asumen la responsabilidad del estudio } \\
\text { (exploración, formulación y validación) } \\
\text { - Se promueve heurísticas y procedimientos personales para resolver problemas }\end{array}$ \\
\hline
\end{tabular}

Fuente: Adaptación de las autoras.

La situaciones-problema que emergen de los contextos propuestos en las macrosituaciones didácticas implementadas, provocan un conflicto cognitivo: por ejemplo, descifrar el enigma de la tabla de doble entrada para averiguar el diseño de las flores que va a llevar la guarda para ambientar la Sala (Tabla 6), leer el diseño del tren a partir un pictograma (Tabla 3) y comprobar la percepción de que "hay muchas casas antiguas que se están arreglando" en las dos cuadras que recorrimos en la primera aproximación al barrio del Jardín en Paisaje Urbano (Tablas 1).

En la puesta en común se confrontan los resultados y puede ocurrir el conflicto socio-cognitivo, por ejemplo, las cantidades y modelos de flores descifradas deberán ser las mismas para algunos grupos de niños que tienen la misma tabla. Las tablas se extrajeron de una caja opaca y había varias réplicas de las mismas tablas. Volver a mirar la tabla ahora, con las otras tablas del grupo, generó ese conflicto socio-cognitivo imprescindible para un aprendizaje con otros y que acompaña el proceso de aprendizaje de cada niño. El grupo, debe arribar a la misma respuesta, porque se tienen la misma información y los modelos de flores son los mismos, así como la cantidad de cada flor.

Se procuran variedad de formas de organización para las tareas emergentes y convergentes a la resolución de las situaciones-problema: la distribución de los niños y niños en las mesas -según el plano de la sala- mediante juegos-experimentos aleatorios sin reposición, grupos pequeños y de manera individual.

Se evalúa el nivel de internalización del contenido curricular desarrollado, por 
ejemplo, la lectura de tablas de doble, está presente en las tres macrosituaciones implementadas, de modo que un mismo contenido se presenta en variados contextos, se reutiliza, resignifica y finalmente internaliza.

\section{CONCLUSIONES}

Consideramos que las Macrosituaciones Didácticas implementadas para la alfabetización estadística en salas de 4 y 5 años tienen un alto grado de idoneidad didáctica global y esto es indicativo de que, enseñamos una estadística potente que niños y niñas aprendieron de manera significativa, situada y lúdica. Ahora bien, debido a que, en Argentina, en el currículo de Matemática de Educación Inicial es casi nula la presencia de los contenidos de Estadística, Combinatoria y Probabilidad resultó necesario que el equipo docente se nutriera de las recomendaciones de la literatura actual relacionada con la alfabetización estadística en la Educación Infantil.

Vale resaltar que, los niños y niñas se mostraron muy interesados en la resolución de las situaciones-problema que emergieron del ambiente natural, social y tecnológico en las tres macrosituaciones implementadas. Asimismo, la dinámica de los tiempos que dan el ritmo al proceso de enseñanza y aprendizaje de Estadística en estas coreografías complejas y enriquecidas, posibilita y promueve la gestión colectiva del conocimiento.

En síntesis y como refiere D'Amore en el prefacio del libro "Matemática con Sentido" (Cabrera y Sosa, 2006, 2008, p.12): "La matemática que los alumnos [niños y las niñas] construyen es una matemática "sana", útil, dotada de sentido".

Estos 21 años de investigación-acción pedagógica continua y situada en las salas del Jardín de Infantes Marqués de Sobremonte permitió germinar una cultura institucional que entiende y vive la alfabetización estadística de las y los niños de manera cotidiana y natural. En acuerdo con Godino (2021) la investigación-acción pedagógica, se considera una estrategia profesional para la mejora e innovación educativa.

Para finalizar, compartimos la reflexión de una de las docentes que estuvo desde el inicio de esta investigación: “El "para qué” me perseguía como un fantasma, la verdad, me quitaba el sueño. ¿Para qué cuento árboles, sillas, puertas, lápices, esto, para qué los cuento? (...) Lo importante es el sentido y comunicar eso que cuento. Aprendí que son datos que usaré para algo, pero ya no quiero que me sirva a mí, ahora quiero que les sirva a ellos, quiero un motivo real, bien cierto. Si la estadística impactó en mi vida, en la de mis estudiantes, bueno que ahora también impacte al pueblo, que ayude para cambiar algo, algo más que mi pensamiento”. 


\section{Agradecimientos}

Agradecemos al comprometido equipo docente del Jardín de Infantes Sobremonte, al equipo de profesoras adscriptas a Matemática y su Didáctica y Ateneo de Matemática de la Práctica IV del Profesorado de Educación Inicial del ISFD "Adoratrices" y estudiantes. Y especialmente, a cada niño y niña que experimentó la alfabetización estadística a sus 4 y 5 años de edad.

\section{REFERENCIAS}

ALSINA, Á., CORNEJO MORALES, C. y SALGADO SOMOZA, M. ¿Cómo, para qué y sobre qué se argumenta en el marco de la probabilidad intuitiva? Un estudio de caso múltiple en Educación Infantil. Revista Paradigma, 12 (1),285-312. 2021

ALSINA, Á. y VÁSQUEZ, C. A. Hacia una enseñanza eficaz de la estadística y la probabilidad en las primeras edades. Didasc@lia: Didáctica y Educación, 8(4), 199-212. 2017.

AROZA, C. J., GODINO, J. D. y BELTRÁN-PELLICER, P. Iniciación a la innovación e investigación educativa mediante el análisis de la idoneidad didáctica de una experiencia de enseñanza sobre proporcionalidad. AIRES, 6 (1), 1-29. 2016.

BATANERO, C. y BOROVCNIK, M. Statistics and probability in high school. Rotterdam: Sense Publishers. 2016.

BATANERO, C. Treinta años de investigación en educación estocástica: Reflexiones y desafíos. En J. M. Contreras, M. M. Gea, M. M. López-Martín y E. Molina-Portillo (Eds.), Actas del Tercer Congreso Internacional Virtual de Educación Estadística. Recuperado 3 de marzo de 2021, de: www.ugr.es/local/fqm126/civeest.html. 2019.

BELTRÁN-PELLICER, P. y GODINO, J. D. Aplicación de indicadores de idoneidad afectiva en un proceso de enseñanza de probabilidad en educación secundaria. Perspectiva Educacional, 56 (2), 92-116. 2017.

BELTRAN-PELLICER, P., GODINO, J. D., \& GIACOMONE, B. Elaboración de indicadores específicos de idoneidad didáctica en probabilidad: aplicación para la reflexión sobre la práctica docente. Bolema: Boletim de Educação Matemática, 32 (61), 526-548. 2018.

BERAZA, M. A. y CERDEIRIÑA, M. A. Coreografías didácticas institucionales y calidad de la enseñanza. Linhas Críticas, 25, 206-221. 2019.

CABRERA, G. y SOSA A. (1ra ed.) Matemática con sentido. Una propuesta que replantea el modo de enseñar la Matemática desde edades tempranas. Córdoba, Argentina: Editorial Comunicarte. 2006.

CABRERA, G. y SOSA A. (2da ed.) Matemática con sentido. Una propuesta que replantea el modo de enseñar la Matemática desde edades tempranas. Córdoba, Argentina: Editorial Comunicarte. 2008.

CABRERA, G. P., TAUBER, L. M. Valoración de la idoneidad didáctica de un proceso de enseñanza para cursos introductorios de estadística en la universidad. En J. M. Contreras, M. M. Gea, M. M. López- Martín y E. Molina-Portillo (Eds.), Actas del Tercer Congreso Internacional Virtual de Educación Estadística. Recuperado de: 
www.ugr.es/local/fqm126/civeest.html. 2019.

CABRERA, G. P., TAUBER, L. M. y FERNÁNDEZ, E. Educación estocástica para pensar estadís-criticamente. Matemáticas, educación y sociedad, 3 (2), 89-109. 2020. Recuperado de: https://www.uco.es/ucopress/ojs/index.php/mes/article/view/12903.

CABRERA, G. P. Coreografías didácticas para la Educación Estocástica en la Educación Inicial y Primaria. II Jornadas de Educación Estadística y I Jornadas Latinoamericanas de Investigación en Educación Estadística. Universidad Nacional de Villa María. 2020. Recuperado de: https://www.youtube.com/watch?v=JfmhNueXIOc.

CID-SABUCEDO, A., PÉREZ, A. y ZABALZA, M. Las prácticas de enseñanza declaradas de los "mejores profesores" de la Universidad de Vigo. RELIEVE, 15 (2), 129. 2013.

CIVArolo, M. y PÉREZ-ADRADA, M. Primeros Pasos en la documentación pedagógica. Argentina: Editorial Hola chicos. 2019.

DE LA BARRERA, M. L., SOMAVILLA, A. R., CHESTA, R. C. y DE PICCOLI, L. Aprender en aulas abiertas: nuevas coreografías didácticas para educadores infantiles. RELAdEI. Revista Latinoamericana de Educación Infantil, 5(4), 173-181. 2016.

DOUADY, R. Relación enseñanza aprendizaje. Dialéctica Instrumento-objeto, juego de marcos. Cuadernos de Didáctica de las Matemáticas, 3. 1984.

FONT V. Los Criterios de Idoneidad Didáctica como guía de la reflexión de profesor sobre su práctica. Conferencia impartida en II Simposio de Educación Matemática (SEM) Virtual. Recuperado el 15 de mayo del 2021, de: https://youtu.be/1PX1WC3WjQI. 2021.

GOBIERNO DE LA PROVINCIA DE CÓRDOBA. MINISTERIO DE EDUCACIÓN Y CULTURA. Dirección de Planificación y Estrategias Educativas. Propuesta Curricular para el Nivel Inicial (1era Versión) Material para la consulta. Córdoba, Argentina. 1998.

GODINO, J. D. Indicadores de la idoneidad didáctica de procesos de enseñanza y aprendizaje de las matemáticas. Cuadernos de investigación y formación en educación matemática, 111-132. 2013.

GODINO, J. D. ¿Cómo salvar la brecha entre la investigación y la práctica en Educación Matemática. Conferencia impartida en II Simposio de Educación Matemática (SEM) Virtual. Recuperado el 15 de mayo del 2021, de: https://youtu.be/1PX1WC3WjQI. 2021.

GÓMEZ, B. R. La investigación-acción educativa y la construcción de saber pedagógico. Educación y educadores, (7), 45-56. 2004.

GONZÁLEZ, A. y WEINSTEIN, E. ¿Cómo Enseñar Matemática en el Jardín?. Argentina: Ediciones Colihue SRL. 1998.

MINISTERIO DE EDUCACIÓN, Presidencia de la Nación, Núcleos de Aprendizaje Prioritarios (NAP), Educación Inicial. 2004. Recuperado de: https://www.educ.ar/recursos/132574/nap-educacion-inicial/download/inline.

MINISTERIO DE EDUCACIÓN, CULTURA, CIENCIA y TECNOLOGÍA, Presidencia de la Nación, Secretaría de Innovación y Calidad Educativa. Indicadores de Progresión de Aprendizajes Prioritarios - IPA 2030. Aprendizajes de Matemática 2030. 2018. Recuperado de:

https://www.argentina.gob.ar/sites/default/files/anexo res cfe 34218 0.pdf. 
OSER, F. K. y BAERISWYL, F. J. Choreographies of teaching: Bridging instruction to learning. Handbook of research on teaching, 4, 1031-1065. 2001.

SANTALO, L. Matemática para no matemáticos. Sociedad Andaluza de Educación. 1990.

SANTALÓ, L. Enfoques. Hacia una didáctica humanista de la Matemática. Buenos Aires, Argentina: Editorial Troquel. 1997.

SOSA, A. Coreografías didácticas para la Educación Estocástica en la Educación Inicial y Primaria. II Jornadas de Educación Estadística y I Jornadas Latinoamericanas de Investigación en Educación Estadística. Universidad Nacional de Villa María. Recuperado de: https://www.youtube.com/watch?v=kIP3shqPTek. 2020.

VÁSQUEZ, C., DÍAZ-LEVICOY, D., CORONATA, C. y ALSINA, Á. Alfabetización estadística y probabilística: primeros pasos para su desarrollo desde la Educación Infantil. Cadernos Cenpec, 8 (1), 154-179. 2018.

VÁSQUEZ, C. Educación Estocástica en el aula escolar: una herramienta para formar ciudadanos de sostenibilidad. Matemáticas, educación y sociedad, 3 (2), 1-20. 2020.

ZABALZA, M. A. Coreografías Didácticas para una enseñanza innovadora. 2017. $\mathbf{2 6}^{\circ}$ Jornadas Internacionales de Educación, Lectura y Educación, una relación que se renueva. [Archivo de video] Recuperado de: https://www.fundacionluminis.org.ar/biblioteca/guia-didactica-la-conferencia-miguelzabalza-beraza-coreografias-didacticas-una-ensenanza-innovadora. 2017. 